\title{
Chiral effective field theory for few-nucleon systems
}

\author{
E. Epelbaum ${ }^{\mathrm{a}}$ \\ ${ }^{a}$ Ruhr-Universität Bochum, Institut für Theoretische Physik II, \\ D-44870 Bochum, Germany
}

Some recent developments in the description of nuclear forces and few-nucleon systems within the effective field theory approach are reviewed.

\section{INTRODUCTION}

Chiral Effective Field Theory (EFT) has become a standard tool for analyzing the properties of hadronic systems at low energy where the perturbative expansion of Quantum Chromodynamics (QCD) in powers of the coupling constant cannot be applied, see [12]. It is based upon the approximate and spontaneously broken chiral symmetry of QCD. Starting from the most general effective Lagrangian for Goldstone bosons (pions in the two-flavor case of $u$ and $d$ quarks) and matter fields (nucleons, $\Delta, \ldots$ ) consistent with the symmetries of QCD, the hadronic S-matrix elements are obtained via a simultaneous expansion in the low external momenta and quark masses. Goldstone boson loops are naturally incorporated and all corresponding ultraviolet divergences can be absorbed at each fixed order in the chiral expansion by the counter terms of the effective Lagrangian.

This perturbative scheme works well in the pion-nucleon and pion-pion sectors, where the interaction vanishes at vanishing external momenta in the chiral limit. In the case of few interacting nucleons one has to deal with non-perturbative problems. Indeed, perturbation theory is expected to fail already at low energy due to the presence of the shallow few-nucleon bound states. A suitable non-perturbative approach has been suggested by Weinberg [3, who showed that the strong enhancement of the few-nucleon scattering amplitude arises from purely nucleonic intermediate states. Weinberg suggested to apply EFT to the kernel of the corresponding scattering equation, which can be viewed as an effective nuclear potential. This idea has been explored in the last decade by many authors. In the following I will briefly address some of the actual topics related to the EFT description of few-nucleon systems.

\section{FEW NUCLEONS AT NEXT-TO-NEXT-TO-LEADING ORDER}

The procedure suggested by Weinberg has been first carried out for two nucleons by Ordóñez and co-workers, who derived a NN potential up to next-to-next-to-leading order (NNLO) in the chiral expansion and performed a numerical analysis of the two-nucleon $(2 \mathrm{~N})$ system [4]. The explicit energy dependence of the effective potential derived in [4] leads, however, to difficulties in applications to $>2 N$ systems but can be eliminated 

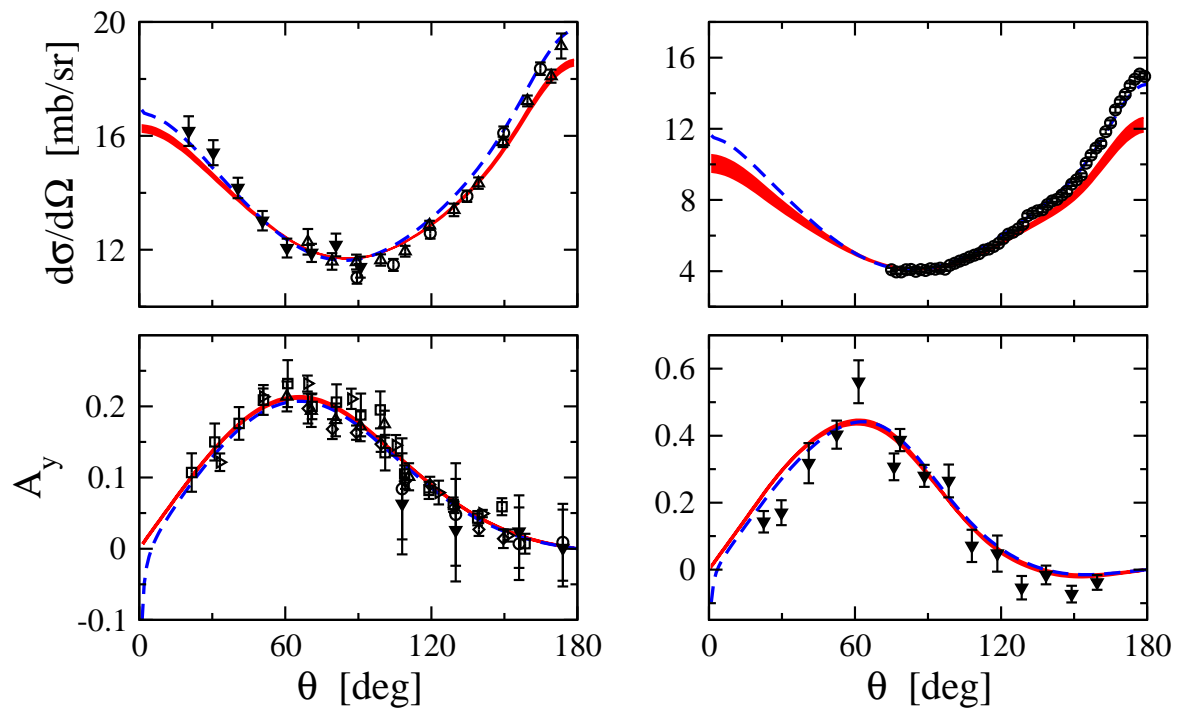

Figure 1. $\quad n p$ differential cross section (upper row) and vector analyzing power (lower row) at $E_{\text {lab }}=50 \mathrm{MeV}$ (left panel) and $E_{\text {lab }}=96 \mathrm{MeV}$ (right panel). Shaded band refers to the NNLO result, dashed line to the Nijmegen phase shift analysis (NPSA) [13]. References to data can be found in [13.

by certain techniques. The corresponding energy-independent expressions for the NN potential have been first given in ref. [5] and later in refs. 67] using different methods. They served as a basis for the NNLO analyses of the two-nucleon (2N) system [89], see also [10] for related application, as well as three- $(3 \mathrm{~N})$ and four-nucleon $(4 \mathrm{~N})$ systems 11] including the corresponding $3 \mathrm{~N}$ forces.

According to chiral power counting the dominant contributions to the effective Hamiltonian for few nucleons are of the order $Q^{0} / \Lambda^{0}$, where $Q \sim M_{\pi}$ refers to the soft scale (typical momenta involved in the process) and $\Lambda$ to the hard scale (chiral symmetry breaking scale, ultraviolet cut-off) [3]. These leading-order (LO) contributions turn out to be of the $2 \mathrm{~N}$ type, given by one-pion exchange (OPE) and two NN contact interactions without derivatives. The first corrections at next-to-leading order (NLO) are of the order $Q^{2} / \Lambda^{2}$ and still of the $2 \mathrm{~N}$ type. They result from two-pion exchange (TPE) with the leading (i.e. with one derivative) $\pi N N$ and $\pi \pi N N$ vertices and 7 independent $N N$ contact interactions with two derivatives. ${ }^{1}$ The only low-energy constants (LECs) entering the expressions for TPE at NLO are the nucleon axial-vector coupling $g_{A}$ and the pion decay constant $F_{\pi}$. Both LECs are measured rather accurately, so that the leading TPE contribution is parameter-free. On the contrary, the LECs accompanying the contact operators are unknown and have been fixed from a fit to low-angular-momentum partial waves.

At NNLO $\left(\sim Q^{3} / \Lambda^{3}\right)$ one has to take into account the subleading TPE contributions given by the triangle diagram with the $\pi \pi N N$ vertex with two derivatives or one insertion of $M_{\pi}^{2}$. The corresponding LECs are denoted $c_{1,3,4}$ and have been fixed in the $\pi N$ system, see e.g. [18. The numerical values of these LECs found in [18] as well as in several other

\footnotetext{
${ }^{1}$ The additional two momentum-independent contact interactions with one insertion of $M_{\pi}^{2}$ can be absorbed by the LO contact operators.
} 

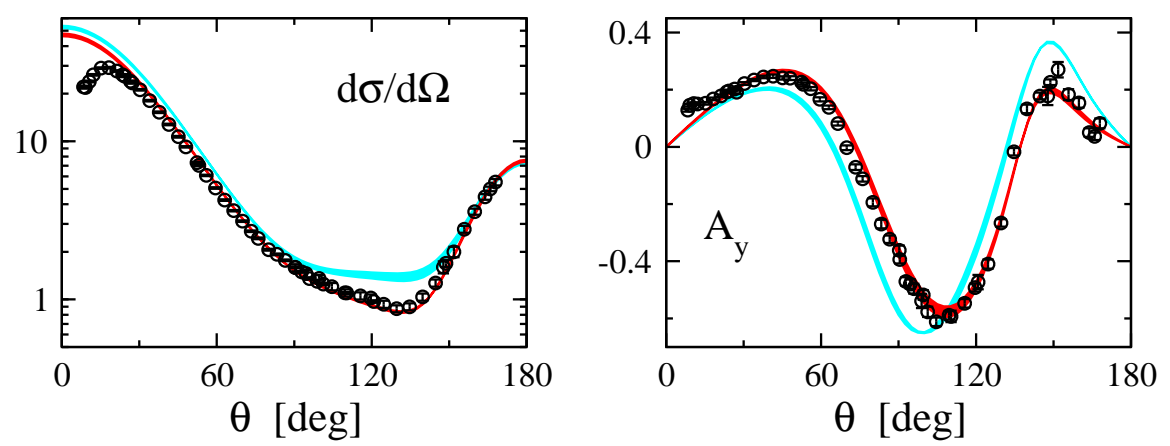

Figure 2. $N d$ differential cross section and vector analyzing power at $E_{\text {lab }}=65 \mathrm{MeV}$. Light (dark) shaded bands refer to the NLO (NNLO) results. Data are from [1415].

analyses of $\pi N$ scattering are rather large compared to what is expected on dimensional reasons, see ref. 9]. Similar large values of $c_{3,4}$ have also been obtained recently from the $n p$ and $p p$ partial wave analysis carried out by the Nijmegen group [19]. The large values of the $c_{3,4}$ can at least be partially explained by the fact that these LECs are saturated by the $\Delta$-excitation [20. This implies that a new and relatively small scale, namely $m_{\Delta}-m_{N} \sim 293 \mathrm{MeV}$ enters the values of these constants in EFT without explicit $\Delta$. The large numerical values of the $c_{i}$ 's lead to the subleading TPE contribution to the NN potential which shows an unphysically strong attraction already at intermediate distances $r \sim 1-2 \mathrm{fm}$ when standard regularization techniques to pion loop integrals (i.e. dimensional or infinite momentum cut-off regularization) are applied. The unphysical behavior of the potential shows up most notably at NNLO in parameter-free predictions for D-waves (F-waves) which start to strongly deviate from the data already at $E_{\text {lab }}>50$ $\mathrm{MeV}\left(E_{\text {lab }}>150 \mathrm{MeV}\right)$ after the subleading TPE contribution is taken into account [6]. Higher order counter terms are needed in order to reduce the strong cut-off dependence of the $\mathrm{D}$-wave phase shifts and to correct the $\mathrm{F}$-waves, which indicates the slow convergence of the chiral expansion. In addition, unphysical deeply bound states arise in low partial waves [8]. Although such deeply bound states do not influence NN observables at low energy, they might lead to certain complications in another processes like e.g. $N d[9]$ and $\pi d$ 21] scattering.

In order to avoid the above mentioned difficulties we have constructed in ref. 9 the NNLO* $^{*}$ version of the potential without deeply bound states. To achieve that, we adopted values of the LECs $c_{3,4}$ much smaller in magnitude than the ones obtained from $\pi N$ scattering. The resulting potential leads to a good description of the low-energy NN data as exemplified with selected observables in Fig. 1.

Lot of progress has also been achieved for $3 \mathrm{~N}$ and $4 \mathrm{~N}$ systems. Scattering and bound state problems in such systems can be solved in a numerically exact way using FaddeevYakubovsky theory and serve as a good testing ground for chiral forces, since most of the unknown parameters are already fixed in the two nucleon system. As already stated before, no $3 \mathrm{~N}$ forces appear at LO and NLO. Parameter-free calculations for $N d$ scattering as well as $3 \mathrm{~N}$ and $4 \mathrm{~N}$ bound states at that order in the chiral expansion have been presented in ref. [16]. We found a good description of various scattering observables at low energy, 
see Fig, The predicted ${ }^{3} \mathrm{H}$ and ${ }^{4} \mathrm{He}$ binding energies

$$
\begin{aligned}
\mathrm{BE}\left({ }^{3} \mathrm{H}\right) & =-7.53 \ldots-8.54 \mathrm{MeV} & & \text { for } \Lambda=500 \ldots 560 \mathrm{MeV}, \\
\mathrm{BE}\left({ }^{4} \mathrm{He}\right) & =-23.87 \ldots-29.57 \mathrm{MeV} & & \text { for } \Lambda=500 \ldots 560 \mathrm{MeV}
\end{aligned}
$$

where $\Lambda$ refers to the momentum cut-off in the $2 \mathrm{~N}$ force, are in a similar range as the ones obtained using modern phenomenological NN potentials and have to be compared with the empirical values $\mathrm{BE}\left({ }^{3} \mathrm{H}\right)=-8.68 \mathrm{MeV}$ and $\mathrm{BE}\left({ }^{4} \mathrm{He}\right)=-29.8 \mathrm{MeV} .{ }^{2}$

Chiral $3 \mathrm{~N}$ forces start to contribute at NNLO and are given by TPE, OPE with the pion emitted (or absorbed) by the NN contact interaction and $3 \mathrm{~N}$ contact interaction. The TPE contribution is parameter-free. The Pauli principle together with the usual symmetry requirements (parity invariance, rotational invariance, ...) lead to a strong reduction of the number of independent terms in the remaining part of the $3 \mathrm{~N}$ force, leaving just one OPE and one contact operator [11. The two corresponding unknown parameters have been fixed from the triton binding energy and nd scattering length, which allowed us to make parameter-free predictions for various other observables. For the $\alpha$-particle binding energy we find at NNLO

$$
\mathrm{BE}\left({ }^{4} \mathrm{He}\right)=-29.51 \ldots-29.98 \mathrm{MeV} \quad \text { for } \Lambda=500 \ldots 600 \mathrm{MeV},
$$

which is rather close to the empirical value. Notice that the cut-off dependence is strongly reduced compared to the NLO result. One also observes improvement for various $3 \mathrm{~N}$ scattering observables when going from NLO to NNLO, see Fig. 2 for two examples.

\section{IMPROVING THE CONVERGENCE OF THE CHIRAL EXPANSION FOR NUCLEAR FORCES}

As already pointed out in the previous section, the numerically large values of the LECs $c_{i}$ found in the $\pi N$ system lead to an unphysically strong attraction of subleading TPE. Kaiser et al. have performed parameter-free perturbative calculations of the peripheral NN partial waves [6] and demonstrated that the nice agreement with the data in D- and F-waves at NLO is destroyed after accounting for the NNLO TPE contribution, which might indicate problems with the convergence of the chiral expansion. Although these problems have been avoided in the previously discussed analysis of the $2 \mathrm{~N}, 3 \mathrm{~N}$ and $4 \mathrm{~N}$ systems by a reduction of the values of $c_{3,4}$, the situation is clearly not satisfactory, since the reduced values of these LECs are not compatible with the $\pi N$ system.

In the following I will explain the origin of the slow convergence and present a new method to improve it. To be specific, consider the isoscalar central part of the subleading TPE which results from the triangle diagram and is given by

$V_{\mathrm{C}}(q)=\frac{3 g_{A}^{2}}{16 F_{\pi}^{4}} \int \frac{d^{3} l}{(2 \pi)^{3}} \frac{l^{2}-q^{2}}{\left((\vec{q}-\vec{l})^{2}+4 M_{\pi}^{2}\right)\left((\vec{q}+\vec{l})^{2}+4 M_{\pi}^{2}\right)}\left(8 c_{1} M_{\pi}^{2}+c_{3}\left(l^{2}-q^{2}\right)\right)$,

where $\vec{q}$ is the nucleon momentum transfer and $q \equiv|\vec{q}|, l \equiv|\vec{l}|$. The integral is cubically divergent and needs to be regularized. Applying dimensional regularization (DR) one finds:

$V_{\mathrm{C}}(q)=-\frac{3 g_{A}^{2}}{16 \pi F_{\pi}^{4}}\left(2 M_{\pi}^{2}\left(2 c_{1}-c_{3}\right)-c_{3} q^{2}\right)\left(2 M_{\pi}^{2}+q^{2}\right) \frac{1}{2 q} \arctan \frac{q}{2 M_{\pi}}+\ldots$

\footnotetext{
${ }^{2}$ These values have been corrected to adjust for $p p$ and $n n$ forces missing in the calculations.
} 

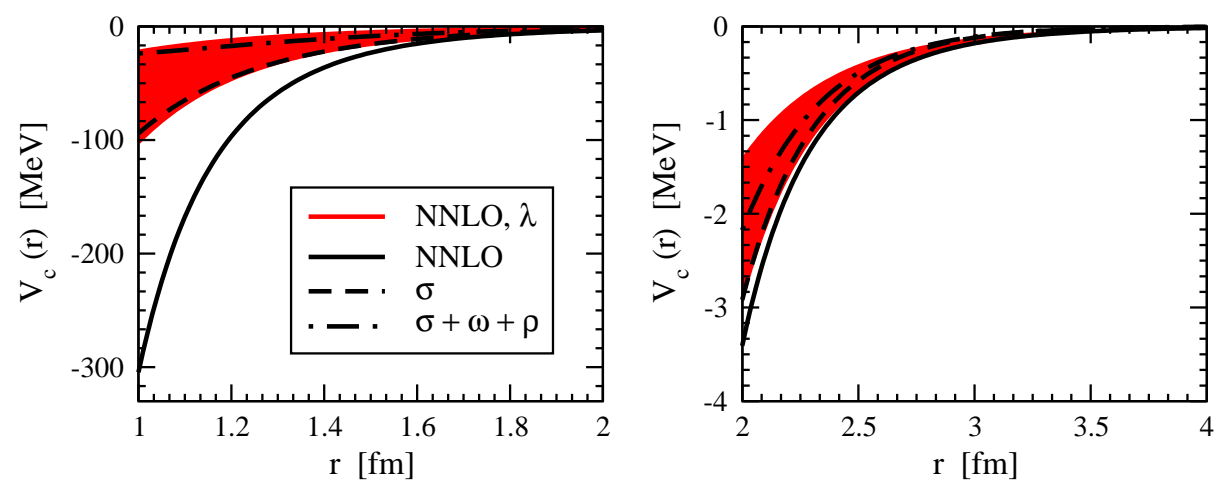

Figure 3. The potential $V_{C}$ in $r$-space. The solid line (shaded band) shows the DR (spectral function regularized, $\lambda=500 \ldots 800 \mathrm{MeV}$ ) result. The dashed (dashed-dotted) line refers to the phenomenological $\sigma(\sigma+\omega+\rho)$ contributions based on the isospin triplet configuration space version (OBEPR) of the Bonn potential [17].

The ellipses refer to polynomial (in $q^{2}$ ) terms of the kind $\alpha+\beta q^{2}$. In this section I will be interested in $\mathrm{D}$ - and higher partial waves, where such terms do not contribute. In order to obtain the potential in coordinate space one has to make an inverse Fourier-transform of $V_{\mathrm{C}}(q)$ in eq. (2). The ordinary inverse Fourier-transform is obviously not possible due to the the fact that $V_{\mathrm{C}}(q)$ growth with $q$. One can nevertheless obtain $V_{\mathrm{C}}(r)$ at each $r>0$ using the spectral function representation [6] :

$V_{\mathrm{C}}(q)=\frac{2 q^{4}}{\pi} \int_{2 M_{\pi}}^{\infty} d \mu \frac{1}{\mu^{3}} \frac{\rho(\mu)}{\mu^{2}+q^{2}}$,

where the spectral function $\rho(\mu)$ can be obtained from $V_{\mathrm{C}}(q)$ in eq. (2) via

$\rho(\mu)=\Im\left[V_{\mathrm{C}}\left(0^{+}-i \mu\right)\right]=-\frac{3 g_{A}^{2}}{64 F_{\pi}^{4}}\left(2 M_{\pi}^{2}\left(2 c_{1}-c_{3}\right)+c_{3} \mu^{2}\right)\left(2 M_{\pi}^{2}-\mu^{2}\right) \frac{1}{\mu} \theta\left(\mu-2 M_{\pi}\right)$.

In eq. (3) the twice subtracted dispersion integral is given which is needed in order to account for the large $-\mu$ behavior of $\rho(\mu)$.

The inverse Fourier-transform in terms of the spectral function $\rho(\mu)$ can easily be evaluated via

$V_{\mathrm{C}}(r)=\frac{1}{2 \pi^{2} r} \int_{2 M_{\pi}}^{\infty} d \mu \mu e^{-\mu r} \rho(\mu)$.

Substituting $\rho(\mu)$ from eq. (41) into eq. (15) and using for the LECs $c_{1,3}$ the central values from ref. 18, $c_{1}=-0.81 \mathrm{GeV}^{-1}$ and $c_{3}=-4.70 \mathrm{GeV}^{-1}$, one obtains the coordinate space representation of the potential $V_{\mathrm{C}}(r)$ shown by the solid line in Fig. 3. The central part of the NNLO TPE potential turns out to be several times more attractive at intermediate distances than the phenomenological $\sigma(\sigma+\omega+\rho)$ contributions. This unphysical attraction shows up in the $\mathrm{D}$ - and $\mathrm{F}$-wave phase shifts as depicted in Fig. 4 , which have been calculated using the Born approximation. While phase shifts at LO (based on OPE) and NLO (based on OPE + leading TPE) are in a fair agreement with the data, NNLO 

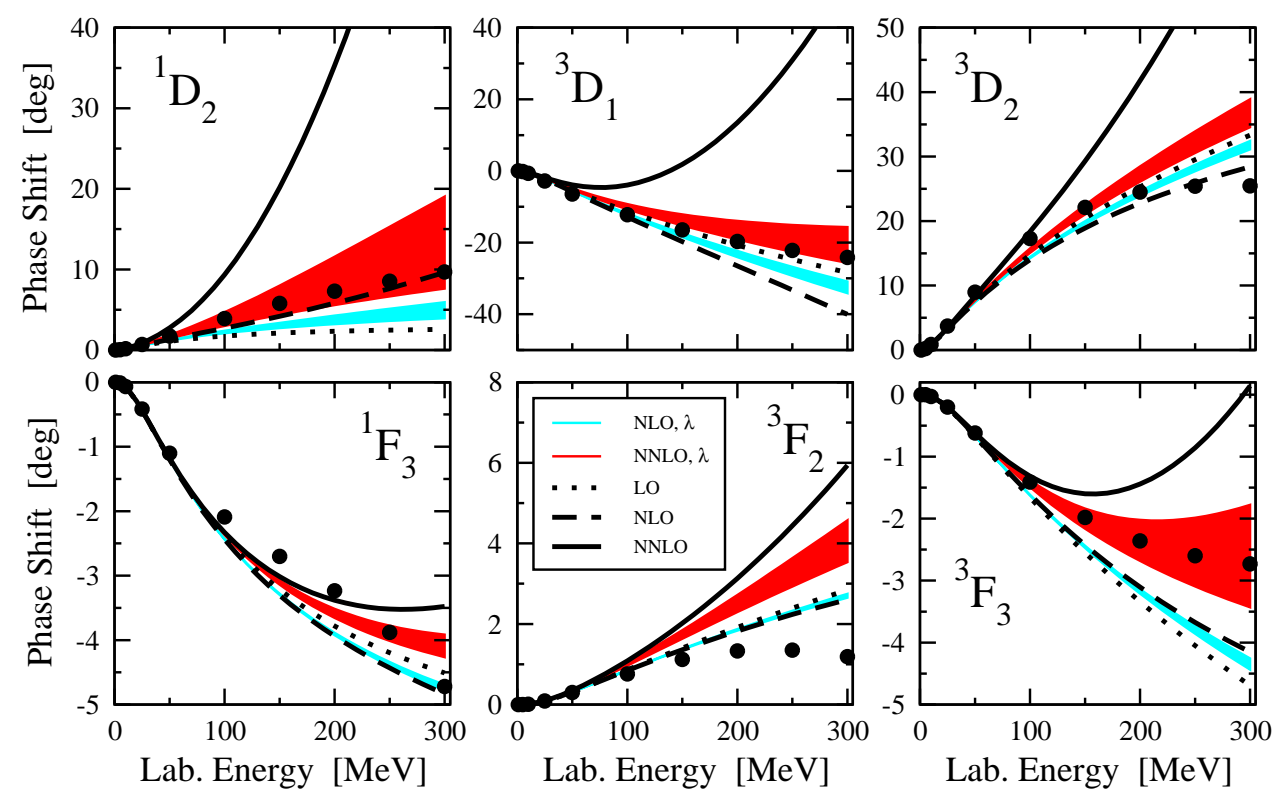

Figure 4. Peripheral NN phase shifts. Dotted line refer to LO, dashed and solid lines (light and dark shaded bands) to NLO and NNLO calculations using DR (the new regularization scheme). Solid dotes are the results from Nijmegen phase shift analysis.

results (based on OPE + leading TPE + subleading TPE) disagree significantly from the data.

The origin of the unphysical attraction at NNLO can be traced back by looking at the integral in eq. (5). In Fig. 5 the integrand in eq. (5) is plotted versus $\mu$ at large $\left(r=2 M_{\pi}^{-1}\right)$, intermediate $\left(r=M_{\pi}^{-1}\right)$ and short $\left(r=0.5 M_{\pi}^{-1}\right)$ distances. While at large distances the integral is dominated by low $-\mu$ (of the order $\mu \sim 350 \mathrm{MeV}$ ) components, already at intermediate distances rather high $-\mu$ (of the order $\mu \sim 600 \mathrm{MeV}$ ) components in the spectral function provide a dominant contribution. Clearly, at shorter distances even higher $-\mu$ components become important. Chiral EFT can hardly provide convergent results for the spectral function at $\mu \sim 600 \mathrm{MeV}$ and higher. Instead of keeping such large$\mu$ components in the regularized loop integral expressions it is advantageous to perform the integration in eq. (5) only over the low $-\mu$ region, where chiral EFT is applicable. This can be achieved by introducing the regularized spectral function

$\rho(\mu) \rightarrow \rho^{\lambda}(\mu)=\rho(\mu) \theta(\lambda-\mu)$,

with the reasonably chosen cut-off $\lambda<M_{\rho}$. Certainly, taking a too small $\lambda$ in eq. (6) will remove the truly long-distance physics while too large values for the cut-off may affect the convergence of the EFT expansion due to inclusion of spurious short-distance physics. Notice that a very similar idea with the finite momentum cut-off has been successfully applied to improve the convergence of the $\mathrm{SU}(3)$ baryon chiral perturbation theory [23 25].

In Fig. 3 we show $V_{\mathrm{C}}(r)$ obtained using the spectral function regularization eq. (6) with $\lambda=500 \ldots 800 \mathrm{MeV}$. The strongest effects of the cut-off are observed at intermediate and short distances, where the unphysical attraction in dimensionally regularized TPE is 
Figure 5. The (normalized) integrand $I(\mu)$ in Eq. (5) for different distances $r$.

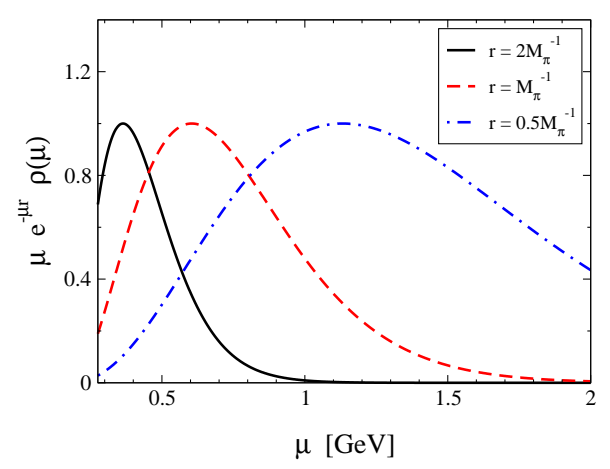

greatly reduced. On the other hand, the asymptotic behavior of the potential at large $r$ is not affected by the choice of regularization. The results for D- and F-waves are greatly improved when the spectral function regularization is used instead of dimensional one, as documented in Fig. 4. The error of about $10(1)^{\circ}$ at $E_{\text {lab }}=300 \mathrm{MeV}$ for the D- (F-) waves appears reasonable.

In ref. 22] we have demonstrated that the spectral function regularization is equivalent to (finite) momentum cut-off regularization of pion loop integrals. It should be understood that this new regularization scheme does not introduce any model dependence in the EFT procedure as soon as $\lambda$ is chosen of the order of (or larger than) $M_{\rho}$. Various choices for $\lambda$ (including $\lambda=\infty$, which is equivalent to dimensional regularization) differ from each other by higher-order contact terms and lead to exactly the same result for observables provided one keeps terms in all orders in the EFT expansion. In ref. [22] we have also demonstrated how to systematically perform renormalization in this scheme, see also [23 24] for the related discussion.

Last but not least, our choice of regularization in eq. (6) is by no means unique. Different choices lead to equivalent results for the potential up to higher order terms and may be used as well. The advantage of the form eq. (6) is that it does not generate spurious longrange contributions which are suppressed by inverse powers of $\lambda[22$. Simple analytical expressions for the regularized loop functions at NNLO are given in ref. [22].

\section{TWO NUCLEONS WITH THE NEW REGULARIZATION SCHEME}

Application of the new regularization scheme to the low partial waves in the nonperturbative regime is the last topic I would like to address. The explicit expressions for the potential up to NNLO derived with the new regularization scheme are given in ref. [26]. In that work the central values from the $Q^{3}$-analysis of the $\pi N$ system [18] have been adopted for the LECs $c_{1,4}: c_{1}=-0.81 \mathrm{GeV}^{-1}, c_{4}=3.40 \mathrm{GeV}^{-1}$. For the constant $c_{3}$ the value $c_{3}=-3.40 \mathrm{GeV}^{-1}$ has been used which is on the lower side but still consistent with the results from ref. [18 $\left(c_{3}=-4.69 \pm 1.34 \mathrm{GeV}^{-1}\right)$. Interestingly, similar values for this LEC have been extracted recently from matching the chiral expansion of the nucleon mass to lattice gauge theory results at pion masses between 500 and $800 \mathrm{MeV}$ [27].

Using the effective potential the bound and scattering states are generated by solving the Lippmann-Schwinger (LS) equation which has to be regularized because of the incorrect behavior of the potential at large momenta (even after removing the large-mass 

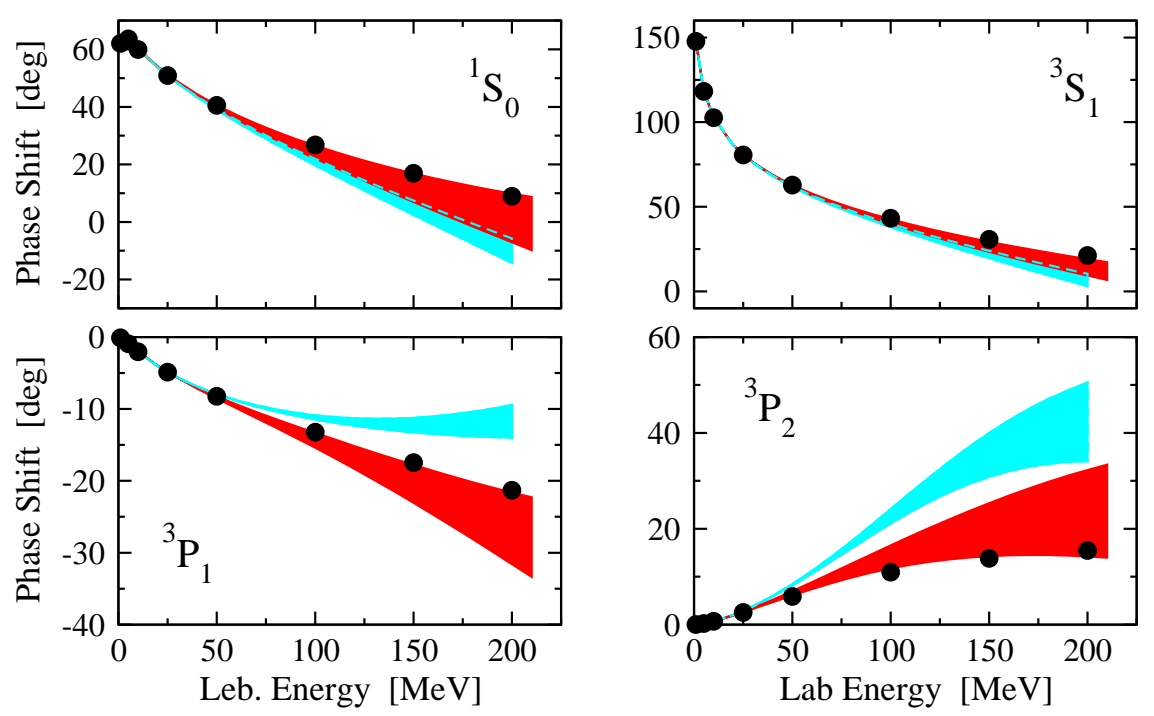

Figure 6. Selected low NN partial waves calculated with the new regularization scheme. For notations see Fig. 4 .

components in the spectrum). We regularize the LS equation in a standard way using the exponential regulator function $f^{\Lambda}(p)=\exp \left[-p^{6} / \Lambda^{6}\right]$, see [26] for more details. In that reference $\Lambda$ has been varied in the range $450 \ldots 600 \mathrm{MeV}$ at NLO and $450 \ldots 650 \mathrm{MeV}$ at NNLO. The cut-off in the spectral function has been varied independently in the range 500. . .700 MeV.

For any choice of $\Lambda$ and $\lambda$ (denoted in ref. [26] by $\tilde{\Lambda}$ ) the 9 LECs accompanying the contact interactions have been fixed from a fit to $n p$ low partial waves at low energy $\left(E_{\text {lab }}<100 \mathrm{MeV}\right)$ in the same way as in [8. Th. This allows to make predictions for higher energies/partial waves. Results for selected partial waves are shown in Fig. 6. The description of the data improves at NNLO compared to NLO in nearly all channels, which is entirely due to inclusion of the subleading TPE contribution. The improvement is also observed for the scattering lengths and effective range coefficients in both $\mathrm{S}$-waves as well as for the deuteron properties [26]. Notice that contrary to the results of ref. [8], where DR has been applied to calculate pion loop integrals, there are now no spurious deeply bound states.

\section{SUMMARY AND OUTLOOK}

I have discussed several topics related to the EFT description of few-nucleon systems. At NNLO, the complete analysis of $2 \mathrm{~N}, 3 \mathrm{~N}$ and $4 \mathrm{~N}$ systems has been performed including for the first time the chiral $3 \mathrm{~N}$ force. Promising results have been obtained at NNLO for various $N d$ scattering observables as well as for the $\alpha$-particle binding energy.

The problem with the unphysically strong attraction of the chiral TPE has been resolved using the new regularization scheme based on the spectral function representation. The method has already been successfully applied to the $2 \mathrm{~N}$ system at NNLO, where it allows to significantly improve the convergence of the EFT expansion. It will be interesting to 
reconsider the $3 \mathrm{~N}$ and $4 \mathrm{~N}$ systems using this formalism.

Finally, it is now of utmost importance to investigate the next order (NNNLO) to be able to make conclusions about the convergence of the chiral EFT for nuclear systems. Work along these lines is in preparation [28] (for a first attempt using dimensional regularization see [29]).

\section{ACKNOWLEDGMENTS}

It is a pleasure to thank my collaborators on these topics, and particularly Walter Glöckle, Hiroyuki Kamada, Ulf-G. Meißner, Andreas Nogga and Henryk Witała.

\section{REFERENCES}

1. U.-G. Meißner, these proceedings.

2. S.R. Beane, these proceedings.

3. S. Weinberg, Phys. Lett. B 251 (1990) 288; Nucl. Phys. B 363 (1991) 3.

4. C. Ordóñez, L. Ray, and U. van Kolck, Phys. Rev. C 53 (1996) 2086.

5. J.L. Friar, and S.A. Coon, Phys. Rev. C49 (1994) 2932.

6. N. Kaiser, R. Brockmann, W. Weise, Nucl. Phys. A625 (1997) 758.

7. E. Epelbaoum, W. Glöckle, and U.-G. Meißner, Nucl. Phys. A637 (1998) 107.

8. E. Epelbaum, W. Glöckle, and U.-G. Meißner, Nucl. Phys. A671 (2000) 295.

9. E. Epelbaum et al., Eur. Phys. J. A15 (2002) 543.

10. D.R. Entem, and R. Machleidt, Phys. Lett. B524 (2002) 93.

11. E. Epelbaum et al., Phys. Rev. C66 (2002) 064001.

12. N. Kaiser, Phys. Rev. C61 (1999) 014003; C62 (2000) 024001; C64 (2001) 057001; C65 (2001) 017001.

13. V.G.J. Stoks et al., Phys. Rev. C48 (1993) 792.

14. S. Shimizu et al., Phys. Rev. C52 (1995) 1193.

15. H. Witała et al., Few-Body Systems 15 (1993) 67.

16. E. Epelbaum et al., Phys. Rev. Lett. 86 (2001) 4787.

17. R. Machleidt, K. Holinde, and Ch. Elster, Phys. Rep. 149 (1987) 1.

18. P. Büttiker, and U.-G. Meißner, Nucl. Phys. A668 (2000) 97.

19. M.C.M. Rentmeester, R.G.E. Timmermans, J.J. de Swart, Phys. Rev. C67 (2003) 044001.

20. V. Bernard, N. Kaiser, and U.-G. Meißner, Nucl. Phys. A615 (1997) 483.

21. S.R. Beane et al., Nucl. Phys. A720 (2003) 399.

22. E. Epelbaum, W. Glöckle, and U.-G. Meißner, Eur. Phys. J. A (2003) in press.

23. J.F. Donoghue, and B.R. Holstein, Phys. Lett. B436 (1998) 331.

24. J.F. Donoghue, B.R. Holstein, and B. Borasoy, Phys.Rev. D59 (1999) 036002.

25. B. Borasoy et al., Phys. Rev. D66 (2002) 094020.

26. E. Epelbaum, W. Glöckle, and U.-G. Meißner, nucl-th/0308010.

27. V. Bernard, Th.R. Hemmert, and U.-G. Meißner, hep-ph/0307115

28. E. Epelbaum, W. Glöckle, and U.-G. Meißner, in preparation.

29. D.R. Entem and R. Machleidt, nucl-th/0304018. 